$\xi=-1$

\title{
Skull Stripping Using Pixel Affinity Graph Method for MRI Head Scans
}

\author{
R.Siva Shankar ${ }^{1 *}$, K.Somasundaram² \\ ${ }^{1}$ Department of Computer Applications, Madanapalle Institute of Technology and Science, Madanapalle-517325,Andhra Pradesh, India \\ ${ }^{2}$ Department of Computer Science and Applications, Gandhigram Rural Institute-DU, Gandhigram-624302,Tamil Nadu, India \\ *Corresponding authorE-mail: arjhunshankar@gmail.com
}

\begin{abstract}
Skull stripping from Magnetic Resonance Image (MRI) of human head scan gives strong impact in clinical diagnosis. The Pixel affinity graph method is used as preprocessing technique, and it is applied on adjacent pixels in each row and column of the middle slice of MRI volume. By grouping the subsets through affinity on intensity found in pixels on the graph (PAG), we can locate the large connected brain portion as subset in the image. After the region of interest is located, Skull is stripped and brain portion is segmented. The proposed PAG based algorithm is validated by comparing the results obtained by the popular automated skull stripping method, Brain Extraction Tool (BET). The qualitative and quantitative results show that the proposed algorithm giving better results.
\end{abstract}

Keywords: MRI; Pixel affinity; Skull Stripping; Segmentation.

\section{Introduction}

Human brain is made up of tissues such as gray matter and white matter and surrounded by cerebra spinal fluid. Magnetic resonance images provides us the maximum extent of information of soft tissues. It is helpful to diagnose the brain related diseases. Segmentation of brain from MRI is performed manually by trained radiologists. Although human vision in natural and easy for limited number of images, but computer mimicry of the human analysis and diagnosis can work for lot of images [1]. Now a days numerous algorithms are employed to segment the brain portion and non-brain region from MR images. Extraction of brain portion is done by methods like region growing and morphological methods. Earlier works on semi automatic methods to segment the brain from MR images have been reported in [2] - [5]. A semi-automatic segmentation method was proposed by Adams et al.[2] required the value of input as the number of seeds for specific regions. Then the formation of regions will be done, finally the expected region will be segmented. A semi-automated segmentation algorithm was proposed by Hohne et al.[3] used region growing method and morphological operations. The segmentation gave 3D visualization with direct visual feedback to guide the user through the segmentation process. Next a semi automatic segmentation method was proposed by Justice et al.[4] using 3D seeded region growing (SRG). This semi-automatic method effectively segments imaging data volumes by having initial seed points and 3D region growing. Another semi-automatic method was proposed by Dubey et al.[5] starts with an intensity-based fuzzy classification of voxels into tumor and background classes. The tumor probability map is used to locally guide the propagation direction and speed of a level-set snake. The tumor probability map is also used to derive an automatic initialization of the snake. This semi automat- ed method give results that have better level of agreement with gold standard than that of automatic methods.

The fully automatic segmentation methods [6-9] improve the speed in obtaining results. The algorithm proposed by Jong and Lee et al.[6] needs histogram analysis to eliminate the background voxels. Then mask is produced by morphological operations. The brain region and non-brain regions were automatically identified by two seeds individually. These seed regions are expanded by $2 \mathrm{D}$ region growing algorithm. The brain anatomy information was used in fixing the seeds for each region. Next automated algorithm was proposed by Lemieux et al.[7] which uses intensity based thresholding technique and morphological operations. The method has been applied on T1-weighted volume to extract human brain from MRI. This three-dimensional method is independent of scan orientation as well.

Stella and Blair et al.[8] proposed an automatic segmentation of brain from MRI. The pipeline of the method includes anisotropic filters, active contouring and prior knowledge of brain anatomy. By those techniques tricky structures like eyes can be removed from brain MRI. This multistage process involved in removal of the background noise from the input image and tracing a rough brain boundary. Then refinement is done to convert the rough brain outline to a final mask. Few of the earlier works [9-11] were done by methods like clustering, resonance principle, Morphology operations, labeling and histogram analysis. All these works are based on the intensity values to do skull stripping. Expectation Maximization (EM) is used in brain segmentation and that was proposed by Pednekar et al., [12] focused on left ventricular (LV) myocardial boundary using intensity based fuzzy affinity method. In that article automatic identification of LV is accomplished by a motion map and segmentation is done by EM algorithm. The au- 
thors have used dynamic programming approach for contour detection.

This paper gives principle used in the research in Section II, Methodology of the work in Section III, Materials used in Section IV, Results and Discussions in Section V, Conclusions of the work and references at last. Proposes a brain extraction scheme using Pixel Affinity Graph method concept for skull stripping in T1 type MRI human head scans.

\section{Principle Used}

Affinity in Graph functions used to relate homogenous objects in matrix with $i, j$ indices. Let $I$ is the input image and the affinity weight matrix $\mathrm{W}$ is obtained using the pixel affinity $(\mathrm{PAI} \rho)$ as :

$$
W_{i, j}=e^{\operatorname{PAI\rho }\left(f_{i}, f_{j}\right)}
$$

The exponential values of each pixel give a better value than the raw PAI $\rho$ values. By observing nearby pixels, we only compute the affinity matrix for pixels within a radius of required pixels from one to another. Remaining affinities are later added or left to 0 , by assuming that different types of features are independent to one another. If we have M subsets of features, then,

$$
W_{i, j}=\int_{k=1}^{M} e^{\operatorname{PAI} \rho\left(f_{i}^{k}, f_{j}^{k}\right)}
$$

The weight matrix $\mathrm{W}$ is calculated for subsets 1 to $\mathrm{M}$. The affinity between the connected pixels reflects different edges. Hence, Pixel affinity calculated for subsets 1 to M. So, similarity between the connected pixels reflects different edges. Each segmented portion will have different weightage due to grouping done by the edges by pixel affinity.

\section{Methodology}

\subsection{Brain boundary detection and extraction in the middle slice}

The middle slice in the volume of each MRI Human head scans contains largest and the connected brain portion compared to the other slices. So we start to calculate pixel affinity value using eqn.(1) for every co-ordinate intensity values in row wise of the middle input image (I). This process is started from the midpoint of the image to the right side boundaries, as well as, mid point to the left boundaries. Computing pixel for row wise produces different edges [13] for the input image. Hence, by applying eq.(2), we obtained the weight matrix which gives the brain boundary (Rbb), around the brain. The flow chart of tracing brain boundary in the middle slice is given in Fig.1.

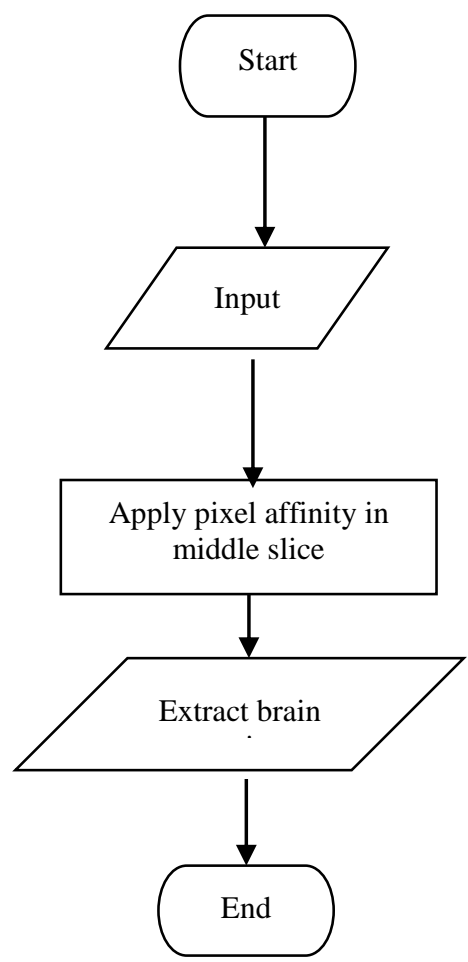

Fig. 1: Flowchart for skull stripping in the middle slice

After segmenting the brain portion from the middle slice, the co-ordinate points of the segmented portion can be used to search the brain portion of the adjacent slices within the co-ordinate points. This will reduce the processing time to find the ROI and produce accurate results.

\subsection{Brain portion Extraction through the Volumes}

The axial brain volume is divided into two sets as upper slices and lower slices. We take the brain portion extracted from middle slice which has brain as a single connected region. This brain region is used as a reference or atlas and processes the adjacent slices lying above and below, in each side parallel and produce the brain mask of each slice. These brain masks are used as atlas to produce the final brain portion of MRI volume.

We start the extraction process at $\mathrm{N}=\mathrm{M} / 2$, where $\mathrm{M}$ is total number of slices in the input volume and $\mathrm{N}$ is the middle slice. Keeping as the co-ordinates of the current slice as reference or atlas, we process the next slice $\mathrm{k}=\mathrm{N}+1$, until $\mathrm{k}=\mathrm{M}$. Only the area of the current slice bounded within the brain portion (BM) extracted from the previous slice is processed and the brain portion is extracted. Similarly, we are doing the similar process which starts from the slice $\mathrm{k}=\mathrm{N}$, and process adjacent slice $\mathrm{k}=\mathrm{N}-1$. This process is repeated until $\mathrm{k}=0$ and the brain portion in each slice are extracted. This makes our process robust. The flowchart of the proposed method through the volumes is shown in Fig.2. 


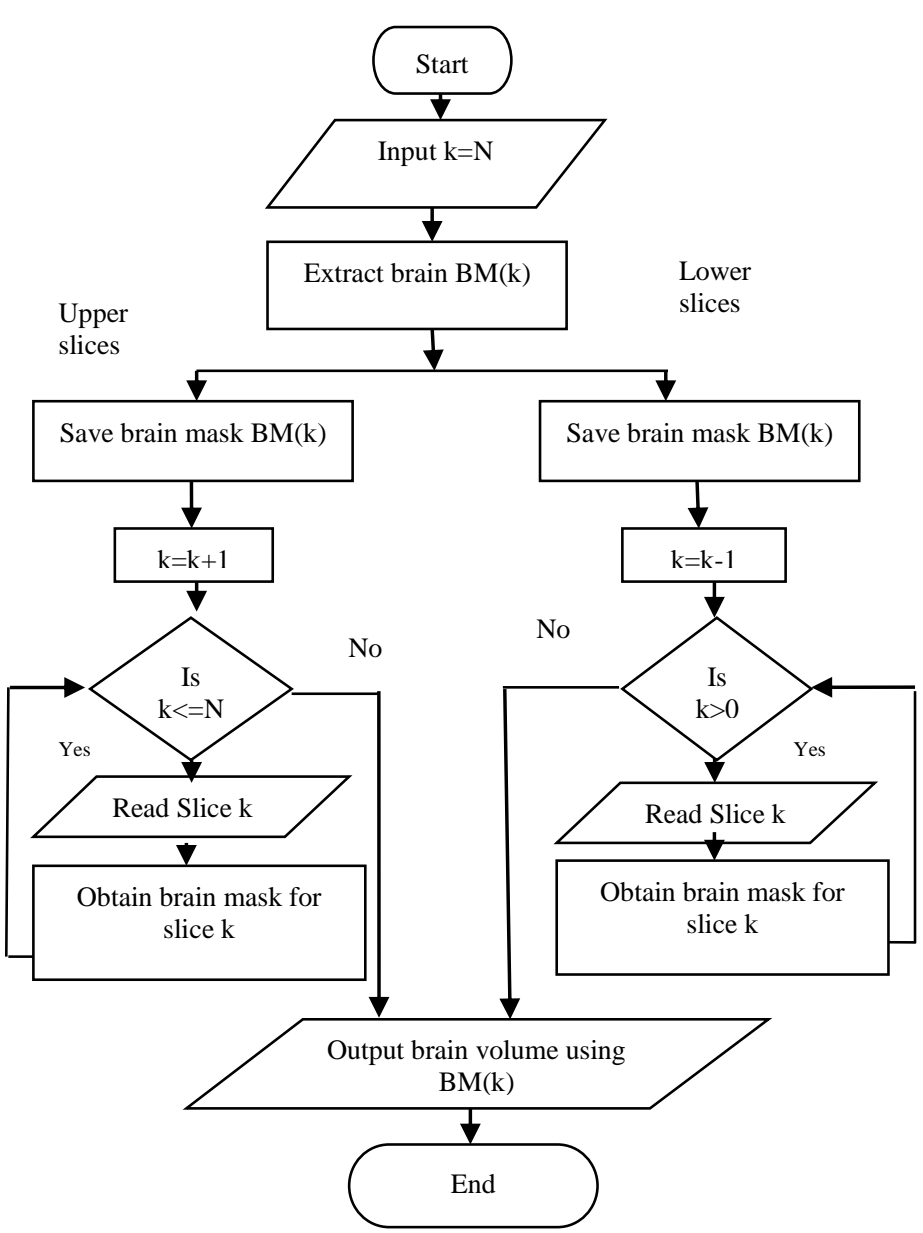

Fig. 2: Flowchart of the proposed method through MRI volumes

\section{Materials Used}

Five T1-weighted axial data sets of MRI were collected from Meenakshi Mission Hospital, Madurai, India [14], for our experiments. The slices were taken from Philips Medical Systems 1.5T machines and $\mathrm{T} 1 \mathrm{w}$ data sets have a dimension of $256 \times 256$ pixels, slice thickness $=5.2 \mathrm{~mm}$ with $6.2 \mathrm{~mm}$ inter-slice gap and number of average $=1$. The field of view is $229 \mathrm{~mm}$, TR $=549 \mathrm{~ms}$, $\mathrm{TE}=14 \mathrm{~ms}$ and flip angle $=73^{\circ}$. The dataset size is nearly 20 slices per volume are common for all the sequences. Medical experts specialized in brain anatomy will manually segment the MRI volumes. The manually segmented slices are considered as gold standard and used to do check similarity measures.

\section{Results and Discussions}

The experiments are carried on the proposed method's five volumes of MRI T1 axial datasets of human head scans by quantitative and qualitative analysis. The evaluation on the performance of the proposed method's results will be compared with manually skull stripped results and the results obtained by of the popular method, Brain Extraction Tool (BET) [15] (Fig.3). For quantitative performance, the parameters Jaccard (J) [16] and Dice (D) [17] were computed using eqn. (3) and (4).

$$
\begin{gathered}
J(A, B)=\frac{|A \bigcap B|}{|A \bigcup B|} \\
D(A, B)=\frac{2|A \bigcap B|}{|A|+|B|}
\end{gathered}
$$

$\mathrm{A}$ and $\mathrm{B}$ in the above equations are two datasets. The value of $\mathrm{J}$ and $\mathrm{D}$ falls from 0 and 1 for disagreement and agreement respectively. The values of $\mathrm{J}$ and $\mathrm{D}$ for BET and our proposed method are recorded and given in Table 1. Fig.4 and Fig.5 shows the plot for the average $\mathrm{J}$ and average $\mathrm{D}$ produced by our method and BET for five volumes. It implies that our method is able to segment brain region completely for five volumes of $\mathrm{T} 1$ axial MR images.

\begin{tabular}{|c|c|c|c|c|}
\hline \multirow{2}{*}{$\begin{array}{c}\text { Da- } \\
\text { ta- } \\
\text { Set }\end{array}$} & \multicolumn{2}{|c|}{ BET } & \multicolumn{2}{|c|}{ Proposed method } \\
\hline & Jaccard & Dice & Jaccard & Dice \\
\hline V1 & .9532 & .9813 & .9573 & .9812 \\
\hline $\mathrm{V} 2$ & .9533 & .9815 & .9598 & .9876 \\
\hline V3 & .9634 & .9796 & .9689 & .9883 \\
\hline V4 & .9542 & .9738 & .9638 & .9872 \\
\hline V5 & .9648 & .9729 & .9612 & .9895 \\
\hline
\end{tabular}

Table 1: The quantitative analysis by Jaccard and Dice coefficients

No

Original Manual

BET Proposed

1

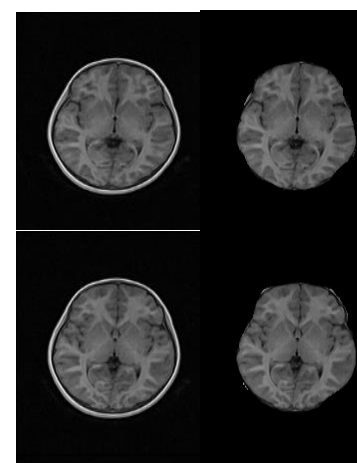

3

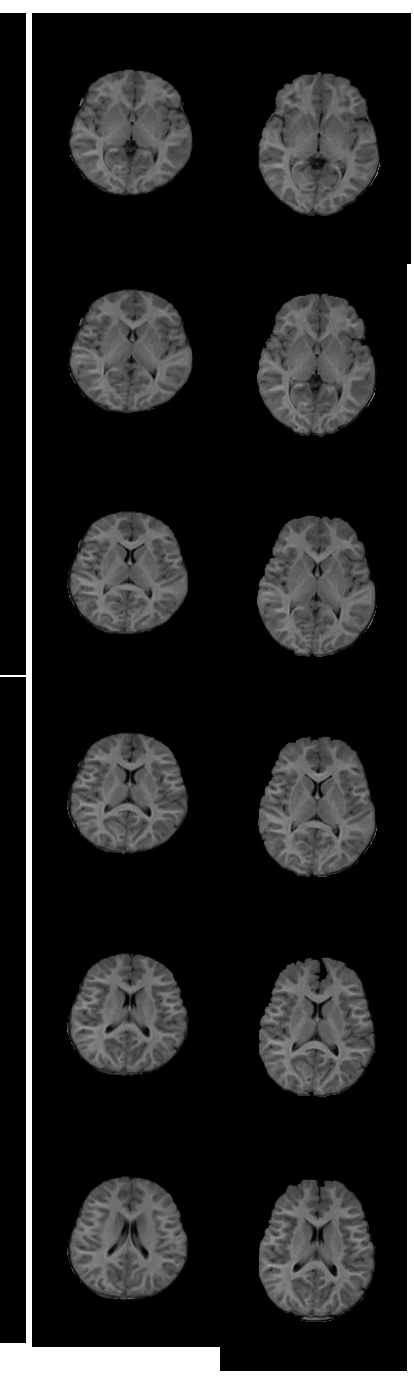

6

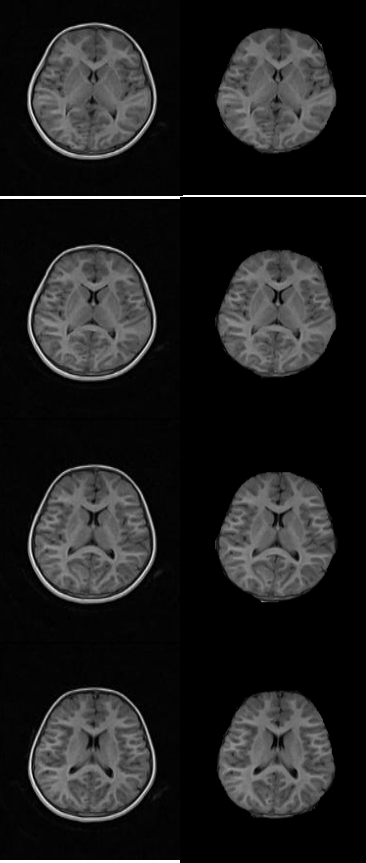

.

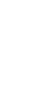

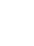


Fig.3 : Original slices and manually segmented slices are shown in first column and second column. The extracted brain images by BET and the proposed method are shown in third and fourth column respectively

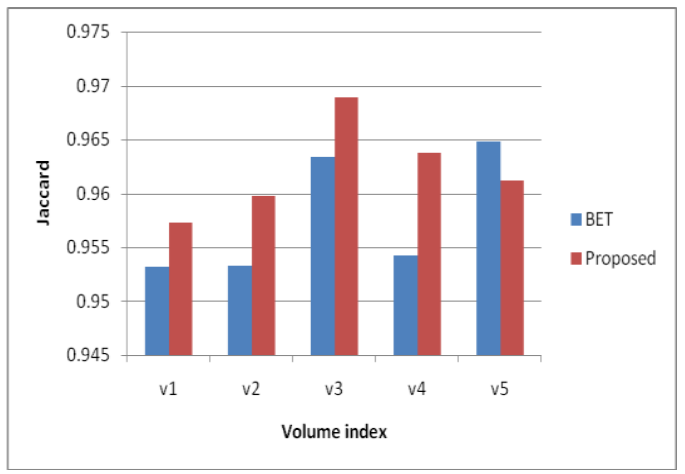

Fig.4 : The average values of Jaccard coefficient $(\mathrm{J})$ computed for $\mathrm{T} 1$ volumes using BET and the proposed method.

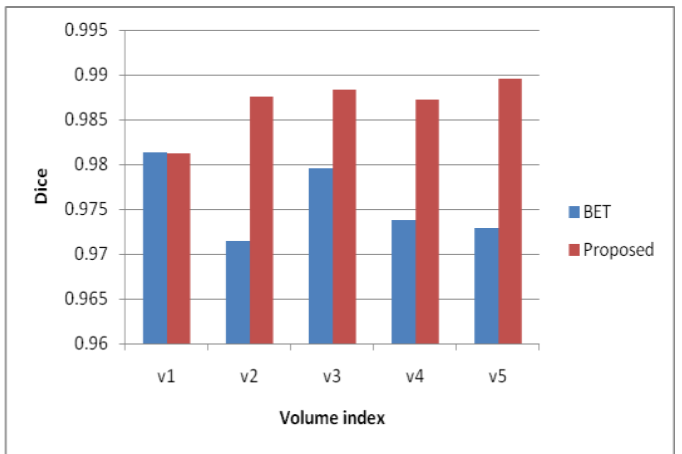

Fig.5: The average values of Dice coefficient (D) computed for T1 volumes using BET and the proposed method

\section{Conclusions}

In this article the Pixel affinity graph method is implemented on adjacent pixels in each row and column of the middle slice of MRI volume. By grouping the subsets by PAG, large brain portion is located. Skull is stripped and brain portion is segmented. The proposed PAG based algorithm is evaluated by comparing the results obtained by the Brain Extraction Tool (BET) and results show that the proposed algorithm giving better results.

\section{References}

[1] Sonka M, Hlavac V, Boyle R, Image Processing, Analysis and Machine Vision, 2nd ed., Thomson Learning (2007), India.

[2]Adams R, Bischof L(1994), Seeded region growing. IEEE Trans. Pattern Anal. Mach. Intell., vol 16, pp.641-646.

[3]Hohne KH , Hanson W A(1992), Interactive 3D segmentation of MRI and CT volumes using morphological operations. J. of Comput.Assist. Tomogr., vol.16, pp. 285-294.

[4]Justice R K, Stokely E M, Strobel J S, Ideker R E, Smith W M (1997), Medical image segmentation using 3D seeded region growing. Proc. SPIE Med. Imag. , vol.3034,pp.900-910.

[5] Dubey R.B., Hanmandlu M., Gupta S.K., and Gupta S.K. (2009), "Semi-automatic Segmentation of MRI Brain Tumor", ICGSTGVIP Journal, vol.9, Issue 4, pp.33-40.

[6]Jong G P, Chulhee L (2009), Skull Stripping based on Region growing for Magnetic resonance brain images. Neurolmage, vol.40, pp.1394-1407.

[7]Lemieux L, Hagmann G, Krakow K, Woermann F G (1999), Fast, accurate, and reproducible automatic segmentation of the brain T1-Weighted volume MRI data. Mgn. Reson. Med., vol.42, pp. 127-135.
[8]Stella Atkins, Blair T Mackiewich (1998), Fully Automatic segmentation of the Brain in MRI. IEEE tracnsations of Medical Imaging, vol.17, pp.98-107.

[9] Somasundaram K, Kalaiselvi T (2011), Automatic brain extraction methods for T1 magnetic resonance images using region labeling and morphological operations. Comput. Bio. Med., vol. 41, pp. 716-725.

[10] Somasundaram K , Siva Shankar R (2012), A novel Skull Stripping Method for T1 Coronal and T2 Axial Magnetic Resonance Images of Human Head Scans Based on Resonance Principle. International conference on Image Processing, Computer Vision and Pattern Recognition organized by WORLDCOMP'12, Las Vegas, Nevada, USA.

[11] Siva Shankar R, Somasundaram K (2017), Automatic Skull Stripping using Maxima-Minima value from Quadratic Equations for MR Images. International Journal for Research in Applied Science \& Engineering Technology, vol.5, pp.647-657.

[12] Pednekar A, Kurkure U, Muthupillai R, Flamm S, Kakadiaris I $\mathrm{A}(2006)$, Automated left ventricular segmentation in cardiac MRI. IEEE Transactions on Biomedical Engineering, vol.53, pp.1425-1428.

[13]Isola P, Zoran D, Krishnan D, Adelson E H(2014), Crisp boundary detection using pointwise mutual information. In European Conference on Computer Vision, Springer, pp.799-814

[14]Madurai Meenakshi Mission Hospitals, Madurai, India.

[15]Smith S M (2002), Fast robust automated brain extraction. Human Brain Mapping, vol.17, pp.143-155.

http://www.fmrib.ox.ac.uk/analysis/research/bet.

[16]Jaccard P (1912), The Distribution of Flora in Alpine Zone. New Phytol., vol.11, pp.37-50.

[17] Dice L (1945), Measures of the Amount of Ecologic Association between Species. Ecology, vol.26, pp.297-302., 1945. 